\title{
Maternal bone mineral changes during pregnancy as measured by peripheral QCT in rural Gambian women with a habitually low calcium intake
}

\author{
M.Ó. Breasail ${ }^{1}$, S. Schoenbuchner ${ }^{1}$, L. Jarjou ${ }^{2}$, S.E. Moore ${ }^{2}$, A. Prentice ${ }^{1,2}$ and K.A. Ward ${ }^{1,4}$ \\ ${ }^{1}$ MRC EWL, 120 Fulbourn Road, Cambridge, CB1 9NL, UK, \\ ${ }^{2}$ MRC Keneba, MRC The Gambia Unit, The Gambia, \\ ${ }^{3}$ Division of Women's Health, Kings College London SE1 7EH and \\ ${ }^{4}$ MRC Lifecourse Epidemiology, University of Southampton, Tremona Rd, Southampton SO16 6 YD
}

Evidence from studies of pre- and post-pregnancy bone densitometry or bone turnover markers suggest maternal bone mineral is mobilised to satisfy the calcium demands of the fetus ${ }^{(1)}$, which at birth contains $25-30 \mathrm{~g}$ Ca. There are now techniques available that can be used during pregnancy to measure changes in the maternal skeleton. We aimed to quantify these changes in a supplementation trial (The Early Nutrition and Immune Development (ENID) trial) that randomized pregnant women before 20 weeks gestation to receive one of 4 supplements daily until term ${ }^{(2)}$. The supplement groups were: control receiving $60 \mathrm{mg}$ iron and $400 \mu \mathrm{g}$ folate as per standard Gambian guidelines, multi-micronutrient (MMN: 15 micronutrients at twice RDA), protein energy (PE: 746 kcal and $20.8 \mathrm{~g}$ protein) or combined PEMMN ${ }^{(2)}$. It was hypothesised that there would be a significant decrease in maternal BMD and structure between early and late pregnancy. We used peripheral QCT to describe these changes and to explore whether supplement type predicted change in bone outcomes.

814 pregnant rural Gambians aged 18-45 years (mean [SD] 29.6 [6.7] years, median parity 4 (IQR 2-6)) were included in this study. Anthropometric and pQCT data at the distal and proximal radius and tibia were obtained at a pre-intervention "booking" visit (mean [SD] 13 [3] wks), week 20, and week 30; (only change between booking and P30 will be described). These pQCT measures were for distal sites: total and trabecular vBMD, total CSA; for proximal sites: total CSA, cortical vBMD, cortical content (BMC), cortical CSA, cortical thickness, and stress-strain index (SSI), an estimate of bone torsional strength. A paired samples t-test was used to determine if change between booking and P30 was statistically significant. Linear regression models tested whether supplement was a significant predictor of change between booking and P30.

No significant differences were observed between booking and P30 in the whole cohort for total vBMD, trabecular vBMD, and total CSA at the either the distal radius or tibia or in total CSA, cortical vBMD, cortical thickness, or SSI at the proximal radius. At the proximal radius there was a small but significant increase in BMC of $0.4 \%(\mathrm{SE} 0.2 \%)$ between baseline and $\mathrm{P} 30$ in the whole cohort $(\mathrm{p}<0.05)$. Cortical vBMD also increased by $0.2 \%($ SE $0.1 \%)(p=0.07)$. A similar pattern was observed at the proximal tibia where increases occurred in parameters of cortical bone, BMC, cortical CSA, and SSI, ranging from $0 \cdot 1-0 \cdot 6 \%($ all $\mathrm{p}<0 \cdot 05$ ). Cortical vBMD also increased $(0.2 \%$ SE $0.1 \%)$, but was not statistically significant $(\mathrm{p}=0.09)$. Supplement type predicted change at the proximal radius where PE supplementation was a significant negative predictor of cortical $\mathrm{BMC}(\mathrm{p}<0 \cdot 05)$ and of cortical $\operatorname{vBMD}(\mathrm{p}<0.001)$. In contrast, PEMMN supplementation was a significant positive predictor of cortical vBMD ( $\mathrm{p}<0.001)$ at the proximal radius. Supplement group was not a predictor of change in any of the outcomes at the distal tibia.

These findings suggest that during pregnancy there is no evidence of bone loss from the trabecular-rich distal radius or tibia and that small increases in cortical bone mineral may occur at the proximal radius and tibia that might be modified by supplementation. The lack of substantial change is somewhat surprising as in lactation release of bone mineral from trabecular sites has been observed consistently and was hypothesised to be the source of bone mineral during pregnancy. Mobilisation of bone mineral may occur in pregnancy through other mechanisms such as changes in maternal trabecular microarchitecture or cortical porosity, however, these are not detectable with pQCT due to limitations of resolution. These may be overcome with the use of more advanced modalities such as high resolution-pQCT.

This research was jointly funded by the Medical Research Council (MRC) (programme codes U105960371, U123261351, MCA760-5QX00) and the Department for International Development (DFID) under the MRC/DFID Concordat agreement.

1. Olausson H, Gail R, Goldberg GR, Laskey MA et al. (2012) Nutr Res Rev, 25, 40-67.

2. Moore SE, Fulford AJC, Darboe MK, et al. (2012) BMC Pregnancy Childbirth 12: 107. 